\title{
Candidate genes for congenital diaphragmatic hernia from animal models: sequencing of FOG2 and $P D G F R \alpha$ reveals rare variants in diaphragmatic hernia patients
}

\author{
SB Bleyl ${ }^{1}$, A Moshrefi ${ }^{2}$, GM Shaw ${ }^{3}$, Y Saijoh $^{4}$, GC Schoenwolf ${ }^{4}$, LA Pennacchio ${ }^{5,6}$ and \\ AM Slavotinek*,2

\begin{abstract}
${ }^{1}$ Division of Medical Genetics, Department of Pediatrics, University of Utah, Salt Lake City, UT, USA; ${ }^{2}$ Division of Genetics, Department of Pediatrics, University of California, San Francisco, CA, USA; ${ }^{3}$ California Birth Defects Monitoring Program, Berkeley, CA, USA; ${ }^{4}$ Department of Neurobiology and Anatomy, University of Utah, Salt Lake City, UT, USA; ${ }^{5}$ US Department of Energy Joint Genome Institute, Walnut Creek, CA, USA; ${ }^{6}$ Genomics Division, Lawrence Berkeley National Laboratory, Berkeley, CA, USA
\end{abstract}

Congenital diaphragmatic hernia (CDH) is a common, life threatening birth defect. Although there is strong evidence implicating genetic factors in its pathogenesis, few causative genes have been identified, and in isolated CDH, only one de novo, nonsense mutation has been reported in FOG 2 in a female with posterior diaphragmatic eventration. We report here that the homozygous null mouse for the Pdgfra gene has posterolateral diaphragmatic defects and thus is a model for human $\mathrm{CDH}$. We hypothesized that mutations in this gene could cause human CDH. We sequenced PDGFR $\alpha$ and FOG2 in 96 patients with CDH, of which 53 had isolated CDH (55.2\%), 36 had CDH and additional anomalies (37.5\%), and 7 had CDH and known chromosome aberrations (7.3\%). For FOG2, we identified novel sequence alterations predicting p.M703L and p.T843A in two patients with isolated CDH that were absent in 526 and 564 control chromosomes respectively. These altered amino acids were highly conserved. However, due to the lack of available parental DNA samples we were not able to determine if the sequence alterations were de novo. For PDGFR $\alpha$, we found a single variant predicting p.L967V in a patient with CDH and multiple anomalies that was absent in 768 control chromosomes. This patient also had one cell with trisomy 15 on skin fibroblast culture, a finding of uncertain significance. Although our study identified sequence variants in FOG2 and PDGFRa, we have not definitively established the variants as mutations and we found no evidence that $\mathrm{CDH}$ commonly results from mutations in these genes.

European Journal of Human Genetics (2007) 15, 950-958; doi:10.1038/sj.ejhg.5201872; published online 13 June 2007

Keywords: congenital diaphragmatic hernia; FOG2; PDGFR $\alpha$; animal models; mutation detection

\footnotetext{
*Correspondence: Dr AM Slavotinek, Division of Genetics, Department of Pediatrics, University of California, 533 Parnassus Street, Room U585P, San Francisco, CA 94143 0748, USA.

Tel: + 1415514 1783;

Fax: + 1415476 9976;

E-mail: slavotia@peds.ucsf.edu

Received 30 January 2007; revised 11 May 2007; accepted 11 May 2007; published online 13 June 2007
}

\section{Introduction}

Congenital diaphragmatic hernia (CDH) is a common birth defect with an estimated prevalence of 1 in 2500 live births. ${ }^{1}$ The prenatal and neonatal mortality for $\mathrm{CDH}$ is high and there is significant long-term morbidity in survivors, including pulmonary hypoplasia, developmental delay, growth retardation and gastro-esophageal reflux. ${ }^{2}$ 
$\mathrm{CDH}$ can occur as an isolated birth defect (isolated CDH) or it can be associated with additional physical anomalies (CDH with additional anomalies or non-isolated $\mathrm{CDH}$ ), either with or without a recognizable underlying genetic syndrome. $^{3}$ In both types of $\mathrm{CDH}$, there is substantial evidence implicating genetic factors in pathogenesis and for genetic heterogeneity, ${ }^{4}$ but there are little data on the causative genes to date. In humans, only one mutation has been demonstrated, occurring in the FOG2 gene in a female patient with posterior diaphragmatic eventration. In non-isolated $\mathrm{CDH}$, several genes have been implicated, but the number of reported mutations remains small.

Gene identification in isolated $\mathrm{CDH}$ has been particularly challenging because the majority of cases are sporadic. One strategy for gene detection utilized array comparative genomic hybridization to identify and define chromosome deletions in patients with $\mathrm{CDH}$ and multiple anomalies. ${ }^{5,6}$ These deleted chromosome regions can be assumed to contain gene(s) necessary for normal diaphragm development, and genes from these 'DH-critical' regions can subsequently be selected for sequencing in isolated $\mathrm{CDH}$ patients. Although several CDH critical regions at $15 \mathrm{q} 26.2$, 8p23.1, 4p16.3 and 1q41-1q42 have been mapped using this strategy, no CDH-causative gene has yet been identified. We have therefore chosen a different approach to study genes potentially causal for human $\mathrm{CDH}$. We have examined mouse models in which diaphragm defects have been observed and focused on two genes-Fog2 and Pdgfr $\alpha$.

The nuclear protein Friend of Gata-2 (Fog2; also known as Zpfm2; OMIM 603693) is a transcriptional repressor with distinct zinc fingers domains that binds to members of the Gata family of transcription factors..$^{7-10}$ FOG2 has been shown to be critical for normal diaphragm development in both mice and humans. Fog2 is expressed in the septum transversum of the diaphragm during early development, and a mouse model with a hypomorphic Fog2 allele has been shown to have diaphragmatic defects and pulmonary hypoplasia. ${ }^{11}$ These mice, referred to as little lung or lil mice, had a splicing mutation with the insertion of $85 \mathrm{bp}$ of intronic sequence into the Fog2 transcript, generating a premature stop codon and a severely truncated Fog2 protein. Homozygous lil mice had diaphragmatic eventration with absence of the musculature of the posterolateral portion of the diaphragm. ${ }^{11}$ In humans, FOG2 has previously been sequenced in 30 patients with diaphragmatic defects. A single, de novo FOG2 mutation, p.R112X, predicting a premature stop codon before the zinc finger domains of the FOG2 protein, was identified in a female with eventration of the posterior part of the diaphragm but not $\mathrm{CDH} .{ }^{11}$ Because it is unclear whether diaphragmatic eventration and hernia are genetically distinct defects in humans, we chose to screen the FOG2 gene for mutations in our larger cohort of patients with diaphragmatic defects.

Platelet-derived growth factor receptor alpha $(P D G F R \alpha$; OMIM 173490) and platelet-derived growth factor receptor beta (PDGFR $\beta$; OMIM 173410) are two tyrosine kinase class III receptors that form homodimers and/or a PDGFR $\alpha \beta$ heterodimer to bind multiple platelet-derived growth factor ligands. ${ }^{12}$ Mice homozygous for null mutations in the Pdgfra gene have a number of genetic backgrounddependent defects, including facial clefting, severe spina bifida occulta, cardiac defects including outflow tract and septal anomalies, omphalocele, renal and urogenital anomalies, vertebral and rib fusion abnormalities, low-set ears and a short neck, short distal limbs and subepidermal blebbing. ${ }^{12-15}$ The requirement for Pdgfr $\alpha$ in lung growth was demonstrated by studies with Pdgfra null mice that also carried a human YAC Pdgfr $\alpha$ transgene. ${ }^{16}$ Many of the $P d g f r \alpha$ null phenotypes were rescued by the transgene allowing assessment of newborn pups. However, the Pdgfr $\alpha$ transgene was not expressed in lung smooth muscle progenitors and the pups died after birth with lung hypoplasia. Our own observations of the Pdgfra null mice, reported below, reveal a spectrum of diaphragmatic defects ranging from absence of the diaphragm to failed or delayed closure of the pleuroperitoneal folds. This murine phenotype shows some overlap with Fryns syndrome (OMIM 229850), a multiple congenital anomaly syndrome in which $\mathrm{CDH}$, pulmonary hypoplasia, cardiac septal defects, cleft palate and lip and dysmorphism are prominent features. ${ }^{17}$ In humans, the PDGFR $\alpha$ gene has been most extensively studied for its role in tumorigenesis. Activating mutations in PDGFR $\alpha$ are common in gastro-intestinal stromal (GIST) tumors ${ }^{18,19}$ and deletions of PDGFR $\alpha$ have been identified in gliomas and glioblastomas. ${ }^{20,21}$ Hypereosinophilic syndrome (OMIM 607685) can result from fusion of the PDGFR $\alpha$ and FIP1L1 genes. $^{22}$ The role of $P D G F R \alpha$ gene in human neural tube defects (NTD) has also been examined, but the association between high-expressing and low-expressing PDGFR $\alpha$ promoter haplotypes and NTD remains controversial. ${ }^{23-25}$ We are not aware that $P D G F R \alpha$ has previously been studied in human $\mathrm{CDH}$, and have not found any data on chromosome rearrangements at the site of this gene at chromosome $4 \mathrm{q} 12$ and human $\mathrm{CDH}$. Because of phenotypic overlap between non-isolated causes of $\mathrm{CDH}$ and the PDGFR $\alpha$ null mouse, and based on the hypothesis that heterozygous mutations in PDGFR $\alpha$ may underlie some cases of isolated $\mathrm{CDH}$, we chose to sequence the PDGFR $\alpha$ gene in our patient cohort.

\section{Materials and methods Patient samples}

DNA samples were obtained from probands and parents under Committee for Human Subjects Research approved protocols at University of California, San Francisco (UCSF). DNA was extracted from peripheral blood lymphocytes, paraffin sections or blood spots obtained from the California Birth Defects Monitoring Program (CBDMP) by digestion with proteinase $\mathrm{K}$ and salting-out according to 
standard procedures. Blood spot DNA was amplified using whole-genome amplification (GenomiPhi ${ }^{\mathrm{TM}}$ GE Healthcare, Piscataway, NJ, USA) according to the manufacturer's instructions and used for DNA sequencing. Phenotypic features were obtained from the CBDMP and tabulated.

\section{Examination of mouse embryo}

The Pdgfr ${ }^{\text {null/null }}$ strain examined in this study (in which exons 2-4 of the gene are deleted) was described previously. ${ }^{12} P d g f r \alpha^{\text {null/ }+}$ mice were mated and pregnant dams killed to collect 12 and $14 \mathrm{dpc}$ embryos under University of Utah IACUC protocol no. 06-04002. The Pdgfr $\alpha$ mutation was examined on a C57BL/6 background. Genotyping was performed using yolk sac samples and multiplex PCR. Pdgfr $\alpha$ mutants are genotyped by mixture of the following three primers: -1,CCCTTGTGGTCATGC CAAAC; -2,GCTTTTGCCTCCATTACACTGG; and -3,CCA ATGGATGAAATCTAAGTGT. Wild-type and null alleles are detected at $450 \mathrm{bp}(1+2)$ and $700 \mathrm{bp}(1+3)$, respectively. Embryos were fixed in 60\% ethanol, 30\% formaldehyde and $10 \%$ glacial acetic acid, embedded in paraffin, sectioned in the transverse plane and stained with hematoxylin and eosin. Serial sections were examined and photographed with a Nikon SMZ1500 stereomicroscope.

\section{Sequencing of FOG 2 and PDGFR $\alpha$ genes}

A high-throughput approach was undertaken as described previously. $^{26}$ Primer oligonucleotides were chosen on repeat-masked DNA using Primer3 (http://frodo.wi.mit. edu/cgi-bin/primer3/primer3_www.cgi), and M13(-21) forward (TGTAAAACGACGGCCAGT) and M13 reverse (CAGGAAACAGCTATGAC) tails were added to each primer. PCR reactions were performed in a 96- or 384-well plate formats using a panel of the human CDH DNAs as template. Amplified PCR products from each individual were sequenced in both directions using BigDye ${ }^{\circledR}$ Terminator v3.1 Cycle Sequencing Kit (Applied Biosystems, Foster City, CA, USA) on an ABI 3730 machine. We sequenced all of the coding exons and exon-intron boundaries of both FOG2 and PDGFRa in all 96 patients, with the exception of the first exon of FOG2, for which we only were able to generate sequence data for 56 patients due to high GC content (data not shown). Sequence variants were identified using the PolyPhred (http:// droog.mbt.washington.edu/PolyPhred/html) or Sequencher (Gene Codes Corporation, Ann Arbor, MI, USA) programs and analyzed manually for accuracy. Sequence variants that were suggestive of mutations were verified by repeat sequencing or restriction enzyme digestion according to standard techniques.

New sequence alterations that were not known polymorphisms in the single-nucleotide polymorphism database (http://www.ncbi.nlm.nih.gov/SNP/) or Ensembl (http://www.ensembl.org/index.html) were verified by repeat sequencing. We also verified three novel alterations not detected in controls (p.M703L and p.T843A in FOG2, and p.L967V in $P D G F R \alpha$ ) in unamplified genomic DNA from the blood spots to exclude the possibility that the nucleotide alterations were introduced by the wholegenome amplification. Control chromosomes were screened for novel sequence alterations by restriction length polymorphism fragment analysis according to standard methods or using a standard Taqman assay. Control samples were obtained from the DNA Polymorphism Discovery Resource (Coriell, Cambden, NJ, USA; http:// ccr.coriell.org/nigms/products/pdr.html); the remainder was control chromosomes collected for other protocolapproved projects. For p.M703L in FOG2, we screened 354 chromosomes from the DNA Polymorphism Discovery Resource and 172 control chromosomes for a total of 526 control chromosomes. For p.T834A in FOG2, we screened 360 chromosomes from the DNA Polymorphism Discovery Resource and 204 control chromosomes for a total of 564 control chromosomes. For p.L967V in PDGFR $\alpha$, we screened 225 individuals of similar ethnicity (African American) and 131 Caucasian and 28 Asian individuals, making a total of 768 chromosomes. Family members were unable to be contacted to verify if alterations were de novo.

\section{Results}

Our observations revealed that $P d g f r \alpha^{\text {null/null }}$ mouse embryos have a range of diaphragmatic defects. We examined diaphragm development at two embryonic stages, 12 and 14 dpc. As described previously, ${ }^{12}$ over half of the null embryos died before $10 \mathrm{dpc}$ based on predicted genotype distributions. Embryos that escape this early lethality and survive to later stages displayed variability in the types and severity of embryonic defects. Of 17 Pdgfr $\alpha^{\text {null/null }}$ mutant embryos scored at $12 \mathrm{dpc}, 7$ (40\%) had diaphragmatic defects ranging from little or no diaphragm formation in severely affected mutants to delayed closure of bilateral posterior pleuroperitoneal folds (Figures 1a and b) in less severely affected embryos. Of the seven Pdgfranull/null mutants surviving to $14 \mathrm{dpc}$, two had open right posterior pleuroperitoneal folds with variable herniation of the stomach into the thorax (Figures 1c and d). Pdgfra ${ }^{\text {null/null }}$ mutants were also noted to have hypoplastic lungs and septal heart defects (Figures 1e and f) as reported previously. These observations establish $P D G F R \alpha$ as a $\mathrm{CDH}$ candidate gene.

Our study cohort consisted of $96 \mathrm{CDH}$ patients, 65 (68\%) had a left-sided diaphragmatic hernia and 22 (23\%) had a right-sided hernia (Table 1). There was one case with absence of the posterior lip of the diaphragm, one patient with a retrosternal hernia and one with eventration (site not specified) and an antral diaphragmatic hernia (1\% each), and the type of diaphragmatic defect was unknown in six patients $(6 \%)$. Fifteen hernias were described as 

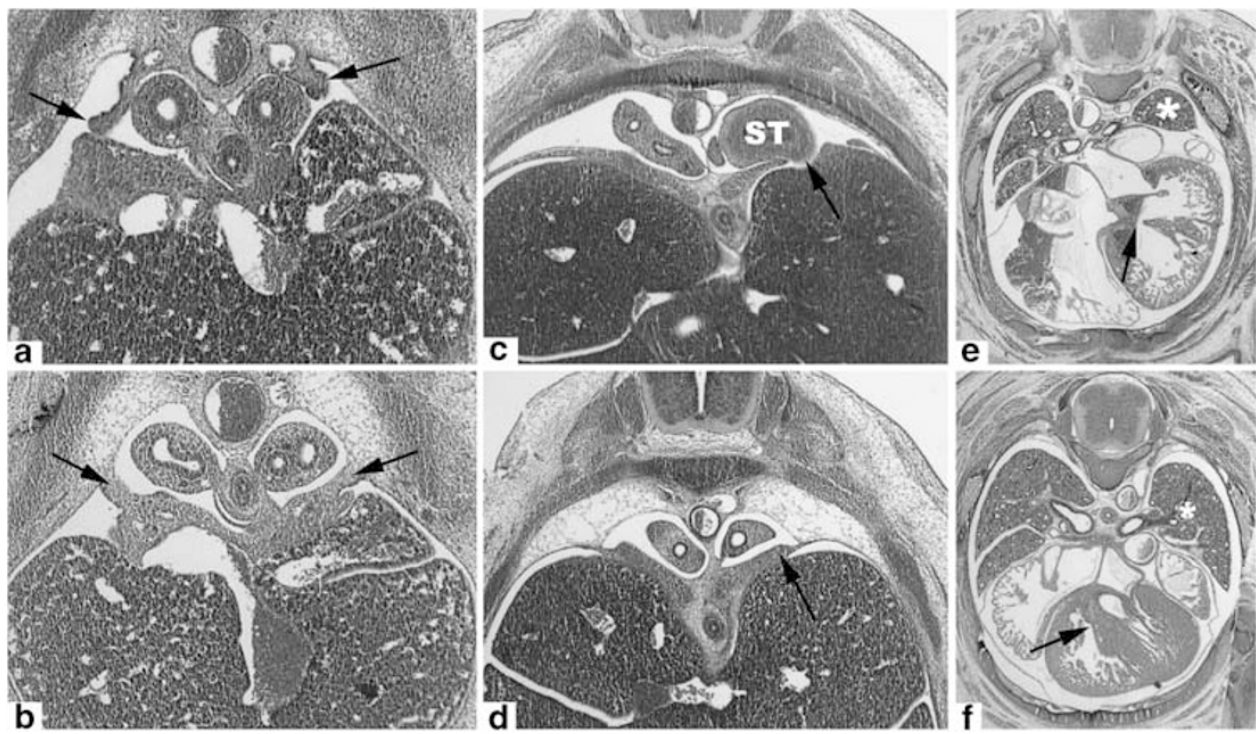

Figure $1 P d g f r \alpha^{\text {null/null }}$ mouse phenotypes. Transverse sections of $12 \mathrm{dpc} P d g f r \alpha^{\text {null/null }}$ embryo (a) showing delayed closure of the pleuroperitoneal folds (PPF; arrows) compared to a Pdgfra null/+ embryo (b). Sections through a $14 \mathrm{dpc}$ Pdgfra ${ }^{\text {null/null }}$ embryo (c) shows failure of the PPF to close (arrow) with herniation of the stomach (ST) into the thorax. This is compared to a Pdgfranul/ + littermate (d) in which the PPF are fused and the stomach is not seen at the same level. Pdgfra ${ }^{\text {null/null }}$ (e) mice were also observed to have pulmonary hypoplasia (asterisk) and septal heart defects (arrow shows a VSD). A $14 \mathrm{dpc}$ Pdgfra ${ }^{\text {null/ }+}$ littermate (f) is shown for comparison.

Table 1 Most common phenotypic features in the $\mathrm{CDH}$ patients

\begin{tabular}{|c|c|c|c|}
\hline Phenotypic feature & Total patients (96) (\%) & Left-sided CDH $(65 ; 68 \%)^{\mathrm{a}}(\%)$ & Right-sided $\mathrm{CDH}(22 ; 23 \%)(\%)$ \\
\hline \multicolumn{4}{|l|}{ Cardiac } \\
\hline Patent foramen ovale & $24(25)$ & $16(25)$ & $7(32)$ \\
\hline Patent ductus arteriosus & $18(19)$ & $13(20)$ & $5(23)$ \\
\hline Tricuspid requrgitation & $30(31)$ & $23(35)$ & $6(27)$ \\
\hline Dextroposition & $13(14)$ & $9(14)$ & $2(9)$ \\
\hline Dextrocardia & $5(5)$ & $4(6)$ & $1(5)$ \\
\hline Mitral regurgitation & $9(9)$ & 9 (14) & $0(0)$ \\
\hline Pulmonary regurgitation & $4(4)$ & $3(5)$ & $1(5)$ \\
\hline Ventricular septal defect & $10(10)$ & $7(11)$ & $2(9)$ \\
\hline Atrial septal defect & $11(11)$ & $9(14)$ & $1(5)$ \\
\hline Pulmonary stenosis & $3(3)$ & $3(5)$ & $0(0)$ \\
\hline Aortic abnormalities $^{b}$ & $3(3)$ & $3(5)$ & $0(0)$ \\
\hline \multicolumn{4}{|l|}{ Pulmonary } \\
\hline Pulmonary hypoplasia & $57(59)$ & $43(66)$ & $11(50)$ \\
\hline CAML & $3(3)$ & $1(2)$ & $1(5)$ \\
\hline \multicolumn{4}{|l|}{ Gastrointestinal } \\
\hline Malrotation & $28(29)$ & $22(34)$ & $4(18)$ \\
\hline \multicolumn{4}{|l|}{ Genitourinary } \\
\hline Renal abnormality/agenesis & $8(8)$ & $7(11)$ & $0(0)$ \\
\hline \multicolumn{4}{|l|}{ Central nervous system } \\
\hline Dandy-Walker malformation & $3(3)$ & $3(5)$ & $0(0)$ \\
\hline Agenesis of the corpus callosum & $2(2)$ & $2(3)$ & $0(0)$ \\
\hline
\end{tabular}

$\mathrm{CDH}$, congenital diaphragmatic hernia; CAML, cystadenomatoid malformation of the lung.

${ }^{a}$ The number in these two columns do not add up to the total number of affected patients, as only patients with left- and right-sided CDH have been listed. The percentages were obtained by dividing the total numbers of patients with left-sided CDH (65) and right-sided CDH (22), respectively.

${ }^{\mathrm{b}}$ Aortic abnormalities include coarctation of the aorta.

Bochdalek in type (16\%) and this description included diaphragmatic defects that involved either the left or the right side.
The most common phenotypic features among these patients are described in Table 1. Cardiac defects were the most frequent, although many of the lesions have been 
reported as pathological changes subsequent to a diaphragmatic hernia, such as patent ductus arteriosus, persistent foramen ovale, dextroposition and tricuspid incompetence. $^{3}$ There was a noticeable phenotypic difference between patients with left-sided $\mathrm{CDH}$ and patients with right-sided $\mathrm{CDH}$, with malformations being more common in patients with left-sided $\mathrm{CDH}$, even when corrected for the greater number of patients with this type of hernia (Table 1), but these differences were not statistically significant. Dysmorphic features and digital anomalies were also more frequently observed in patients with leftsided CDH compared to right-sided $\mathrm{CDH}$ (data not shown).

The results from the FOG2 gene sequencing are shown in Table 2 and the results from the PDGFR $\alpha$ gene sequencing are shown in Table 3. Chromatographs with the sequence alterations are shown in Figure 2. Figure 3 shows the location of sequence variants within the FOG2 and PDGFR $\alpha$ genes with relation to the encoded proteins, and provides amino acid alignments of the affected protein regions among multiple species.

For FOG2, we detected two sequence variants in $\mathrm{CDH}$ patients that were not present in control chromosomes, c.A2107C, predicting p.M703L, and c.A2527G, predicting p.T843A (Table 2). These sequence variants were not found in 526 and 564 control chromosomes, respectively. Both of these alterations were present in exon 7 of the gene. Although these sequence variants result in conservative amino acid substitutions, they occur at conserved sites (Figure 3) and in or near putative zinc finger domains of the protein (amino acids 687-710 and 854-877; http:// ca.expasy.org/).

For $P D G F R \alpha$, we found one novel sequence variant, c.C2889G, predicting p.L967V (Table 3). This change was not seen in 225 control individuals of similar ethnicity (African American), nor in 131 Caucasians or in 28 Asians, making a total of 768 chromosomes. The variant occurs in a domain of the protein conserved in all higher vertebrates (Figure 3), but outside the tyrosine kinase domains (amino acids 593-954; http://ca.expasy.org/). This patient had leftsided $\mathrm{CDH}$ with multiple anomalies comprising pulmonary hypoplasia, congenital heart disease with a ventricular septal defect, secundum type atrial septal defect and tricuspid regurgitation, malrotation of the intestines, a multicystic liver with an abnormal biliary tree, a short neck with slight webbing, a small chest cavity with abnormal thoracic vertebrae, including butterfly vertebrae, and multiple rib anomalies, dysmorphism with a prominent, sloping forehead, small and downslanting palpebral fissures with sparse eyebrows, a flat and broad nasal root, low-set ears that had thick, protruding helices, bilateral simian creases, fifth finger clinodactyly, adducted thumbs and streaky hyper and hypopigmentation of the skin. A skin karyotype showed 46,XX with $1 / 50$ cells showing mosaicism for trisomy 15 . This level of mosaicism could represent an artifact of the fibroblast culture, as there was

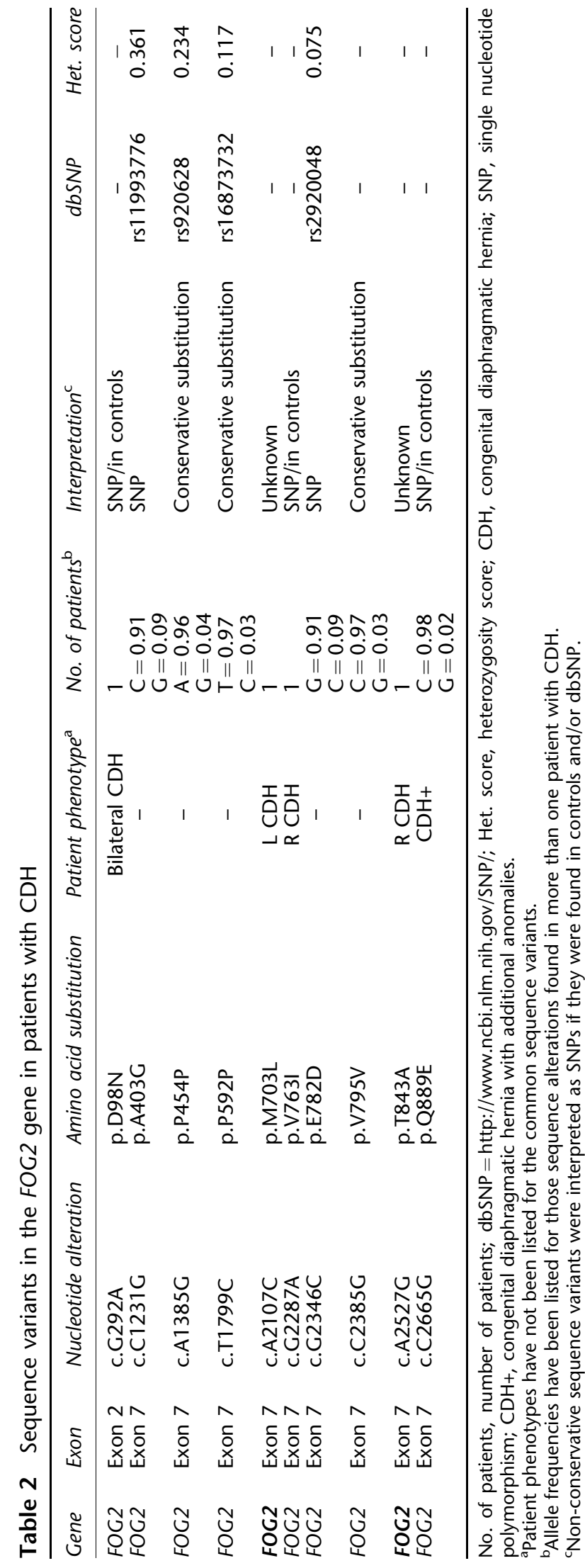


Table 3 Sequence variants in the PDGFR gene in patients with congenital diaphragmatic hernia

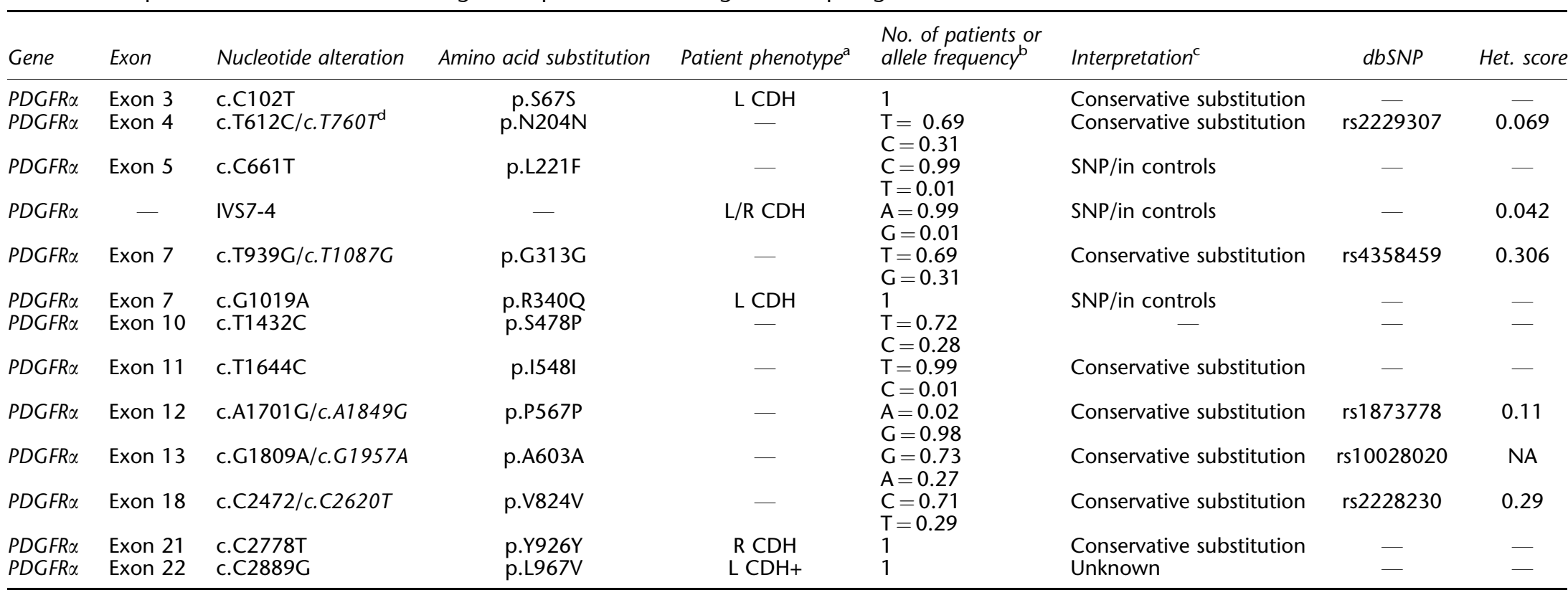

No. of patients, number of patients; dbSNP, http://www.ncbi.nlm.nih.gov/SNP/; Het. score, heterozygosity score; CDH, congenital diaphragmatic hernia; SNP, single nucleotide polymorphism; $\mathrm{CDH}+$, congenital diaphragmatic hernia with additional anomalies.

a Patient phenotypes have not been listed for the common sequence variants.

${ }^{b}$ Allele frequencies have been listed for those sequence alterations found in more than one patient with CDH.

'Non-conservative sequence variants were interpreted as SNPs if they were found in controls and/or dbSNP.

${ }^{d}$ First nucleotide position according to start codon $A(T G)=1$; second nucleotide position according to dbSNP (italicized). 


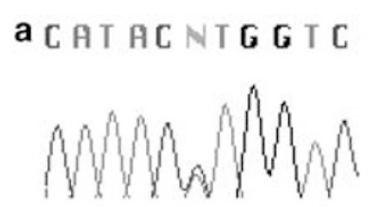

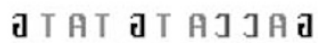

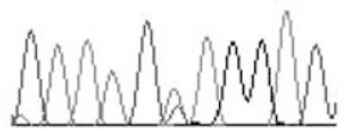

b HG L G G N E G HE L H I

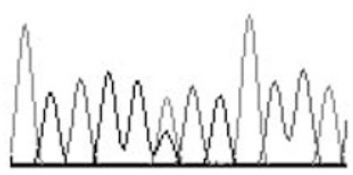

I Jd」Jj」ld d I

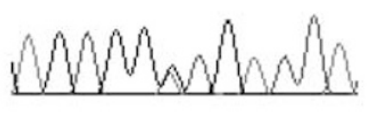

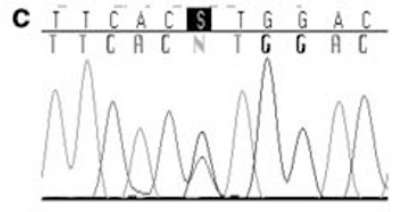
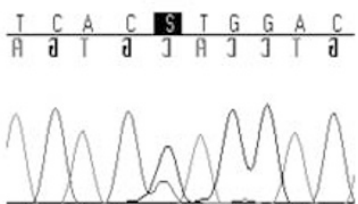

Figure 2 (a) Forward and reverse chromatograms showing c.A2107C, predicting p.M703L in the FOG-2 gene from a patient with a left-sided CDH. (b) Forward and reverse chromatograms showing C.A2527G, predicting p.T843A in the FOG-2 gene from a patient with a right-sided CDH. (c) Forward and reverse chromatograms showing c.C2889G, predicting p.L967V in the PDGFR gene from a patient with a left-sided CDH and multiple anomalies.

only one mosaic cell. However, as discussed below, there is overlap between the phenotype of this patient with characteristics seen in cases of trisomy 15 mosaicism. Conversely, there is also phenotypic overlap between the patient and Pdgfra null mouse embryos. The $\mathrm{CDH}$ in our patient could be related to the trisomy 15 mosaicism, the PDGFR $\alpha$ alteration or a combination of both. However, diaphragmatic defects have not been described with trisomy for any part of chromosome 15.

\section{Discussion}

We selected two genes for screening in human patients with isolated $\mathrm{CDH}$ or $\mathrm{CDH}$ with additional anomalies based on selected animal models of abnormal diaphragm development. Our strategy was successful in identifying sequence variants that were not detected in controls in both of these genes, FOG2 and PDGFR $\alpha$. However, we detected only three new sequence variants, implying that neither of these genes is a major contributor to the pathogenesis of $\mathrm{CDH}$. In addition, we could not determine if the sequence variants were de novo in our patient cohort. We consider that haploinsufficiency for the gene product is the mechanism responsible for the diaphragmatic defects for the sequence variants in the FOG2 and PDGFR $\alpha$ genes, and we identified only one variant in each patient.

The mechanism for the diaphragmatic defects in FOG2 null mice and lil mice with a splicing defect in FOG2 has not been determined. Whole-mount studies showed that the myotubules of the mutant lil diaphragms radiated in a dorsal ventral pattern rather than the mediolateral pattern of wild-type mice and in situ hybridization studies in the lil mice documented reduced expression of the $\mathrm{Hgf} / \mathrm{s} f$ (Hepatocyte growth factor/scatter factor) gene in the part of the diaphragm where the pleuroperitoneal folds meet the septum transversum. ${ }^{11}$ The $H g f / s f$ gene is involved in the migration of muscle cell precursor cells to the diaphragm, and this gene has previously been implicated in diaphragm formation, as mice with absence of the $\mathrm{Hgf} / \mathrm{sf}$ receptor, cmet, are unable to muscularize the diaphragm. ${ }^{27}$ It has therefore been hypothesized that Fog 2 may be required for the induction of $H g f / s f$, and the eventration phenotype of the lil mice could be caused by dysregulation of $\mathrm{Hgf} / \mathrm{sf}$ expression along the path of muscle cell precursor migration between the pleuroperitoneal folds and the diaphragm. ${ }^{11}$

There is additional evidence that FOG2 is critical for normal diaphragm formation. FOG2 was shown to be an important regulator of Gata-4 in the developing heart, and both genes are co-expressed during cardiac embryogenesis. $^{28}$ Interestingly, $\mathrm{C} 57 \mathrm{Bl} / 6$ mice that were heterozygous for a deletion mutation of the Gata-4 gene have midline diaphragmatic defects, ${ }^{29}$ suggesting that abnormal regulation of Gata- 4 by FOG 2 might also be important for diaphragm development. FOG2 also binds to the ligandbinding domain of chicken ovalbumin upstream promoter transcription factor II (COUP-TFII; also known as NR2F2). ${ }^{30}$ COUP-TFII has been shown to be necessary for FOG2 to repress the transcription of a Gata- 4 p.E215K mutant that is unable to bind FOG2, demonstrating that COUP-TFII is necessary for the FOG2-mediated repression of GATA-4 transcription. $^{30}$ Interestingly, COUP-TFII is located on human chromosome $15 \mathrm{q} 26.2$, a genomic region that has been deleted in $\mathrm{CDH}$ patients, ${ }^{31}$ although mutations in this gene have not been demonstrated in human $\mathrm{CDH}$ patients.

In one patient with $\mathrm{CDH}$ and multiple anomalies, sequencing of the $P D G F R \alpha$ showed a missense alteration that predicted p.L967V. However, this patient also had a very low level of mosaicism for trisomy 15 with one trisomic cell out of 50 cells counted on a skin fibroblast culture. Trisomy 15 mosaicism has been associated with developmental delay, congenital cardiac disease, lung hypoplasia, rib anomalies, facial dysmorphism with a broad nasal bridge, an anteverted and prominent nose, a small mouth, narrow lips, ear dysplasia, 
micrognathia, digital anomalies, a high or cleft palate and a short neck. ${ }^{32}$

The physical anomalies in our patient could be related to the trisomy 15 mosaicism, the PDGFR alteration or a combination of both. While the low level of mosaicism in

a

FOG2 gene variants.

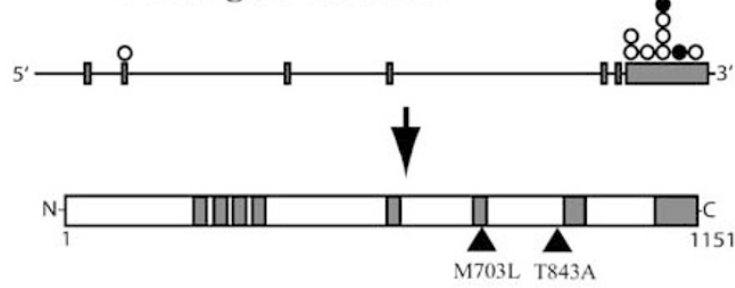

\begin{tabular}{|c|c|}
\hline \multicolumn{2}{|c|}{ p.M703L Variant } \\
\hline Human & NITFSRHETYMVHKQYYCATR \\
\hline Chimp & NITFSRHETYMVHKQYYCATR \\
\hline Mouse & NITFSRHETYMVHKQYYCATR \\
\hline Rat & NITFSRHETYMVHKQYYCATR \\
\hline Dog & NITFSRHETYMVHKQYYCATR \\
\hline Cow & NITFSRHETYMVHKQYYCATR \\
\hline Opossum & NITFSRHETYMVHKQYYCATR \\
\hline Chicken & NITFSRHETYMVHKQYYCATR \\
\hline Conserved & 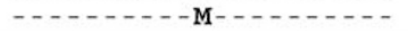 \\
\hline
\end{tabular}

\section{p.T843A Variant}

Human

SKKCLSQSERTTTSPKRLLDY

Chimp

SKKCLSQSERTTTSPKRLLDY

Mouse

SKKCLSQSERTTTSPKRLLDY

Rat

Dog

SKKCLSQSERTTASPKRLLDY

Cow

SKKCLSQSERTTASPKRLLDY

Opossum

SKKCLSQSERTTTSPKRLLDY

Chicken

SKKCLSQSERTTTSPKRLLDY

Conserved

PKKCLSQSERTSTSPKRLLDY

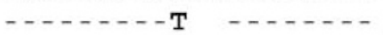

b PDGFRA gene variants.

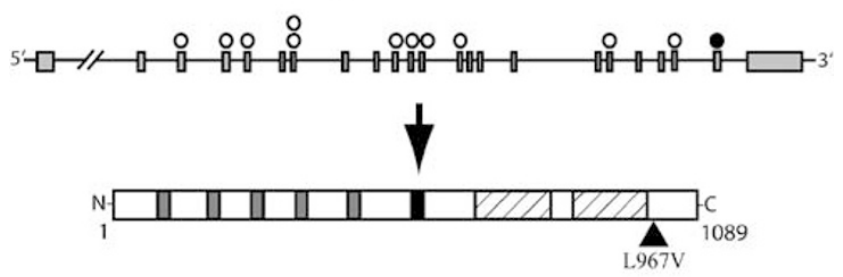

\begin{tabular}{|ll|}
\hline p.L967V Variant & \\
Human & KSYEKIHLDFLKSDHPAVARM \\
Chimp & KSYEKIHLDFLKSDHPAVARM \\
Mouse & KSYEKIHLDFLKSDHPAVARM \\
Rat & KSYEKIHLDFLKSDHPAVARM \\
Dog & KSYEKIHLDFLKSDHPAVARM \\
Cow & KSYEKIHLDFLKSDHPAVARM \\
Opossum & KSYEKIHLDFLKSDHPAVARM \\
Chicken & KSYEKIHLDFLKSDHPAVTRM \\
Conserved & $-\ldots-\ldots-\ldots-$ L_-..-. - - \\
\hline
\end{tabular}

this patient could represent lab artifact, it is also possible that the level of mosaicism was higher in vivo than in culture. It is well known that there is no reliable correlation between mosaicism level and phenotype. Other factors in favor of trisomy 15 mosaicism as a causative factor in this patient include the streaky skin pigmentary changes, which are common in chromosomal mosaicism, and the finding of several anomalies that have previously been described in other patients with mosaicism for trisomy 15 , such as a cardiac defects, a short and webbed neck, rib anomalies and craniofacial dysmorphism including dysplastic ears. However, to our knowledge, diaphragmatic defects have not been described in patients with trisomy for chromosome 15. Our patient also shares many phenotypic features seen in Pdgfra null mouse embryos including $\mathrm{CDH}$.

In mouse studies, $P d g f r \alpha$ has been shown to be critical for the normal embryonic development of multiple organ systems, and our observations add the diaphragm to the list of structures that develop abnormally in the absence of this gene. Combining our finding of diaphragmatic defects and other points of phenotypic overlap with the human Fryns syndrome, we considered the Pdgfr $\alpha$ gene a candidate for human $\mathrm{CDH}$ with or without other anomalies. The specific role of PDGF signaling in diaphragm development and the mechanisms leading to diaphragm abnormalities in these mutants is difficult to determine due to their limited viability. The expression of $P D G F R \alpha$ in the developing diaphragm or diaphragm precursors has not been reported and was not addressed by our study. Future studies using conditional mutations of the mouse Pdgfr $\alpha$ gene and diaphragm-specific Cre driver lines, thus avoiding the high rates of embryonic lethality, could provide more information about the role of this gene in diaphragm development.

\section{Conclusion}

We have identified a novel sequence variant in the PDGFR $\alpha$ gene in an individual with $\mathrm{CDH}$ and multiple anomalies who also had one cell with trisomy 15 on skin fibroblast

Figure 3 (a) The FOG 2 gene is composed of 7 exons and spans $485 \mathrm{~kb}$. It encodes an 1151 amino acid zinc finger DNA binding protein. Eight single-nucleotide polymorphisms or synonomous variants (open circles) were found. Two rare variants, p.M703L and p.T834A (black circles, black triangles), were found in exon 7 and lie near putative zinc finger domains (indicated by gray boxes). Alignment of amino acid residues adjacent to p.M703L and p.T834A shows conservation among different species. (b) The PDGFR gene is composed of 23 exons and spans $70 \mathrm{~kb}$. It encodes a 1089 amino acid receptor tyrosine kinase with five extracellular immunoglobulin domains (gray boxes), a transmembrane domain (black box) and two intracellular tyrosine kinase domains (hatched boxes). Eleven single-nucleotide polymorphisms or synonomous variants (open circles) were found in coding regions. The p.L967V variant (black circle, black triangle) lies downstream of the tyrosine kinase domains. Alignment of amino acid residues adjacent to p.L967V shows conservation among different species. 
culture, suggesting that rare variants in this gene may contribute toward the pathogenesis of $\mathrm{CDH}$. We also identified two novel sequence alterations in FOG2 in two patients with CDHs, further suggesting a role for sequence variants in this gene in the pathogenesis of diaphragmatic hernia in addition to diaphragmatic eventration.

\section{Acknowledgements}

The Pdgfr $\alpha^{\text {null } /+}$ mouse strain was kindly provided by Philippe Soriano. Anne Slavotinek was generously funded by an R03 grant 5R03 HDO49411-02 from the National Institute of Child Health and Development (NICHD) and a Hellman Award (Hellman Family Trust). Steven Bleyl is supported by the Clinical Genetics Research Program at the University of Utah, the Primary Children's Medical Center Foundation, and NIH grant 1K08HL084559. Gary Schoenwolf is supported by NIH grants DC04185, DK066445 and DK065941. Research conducted at the EO Lawrence Berkeley National Laboratory and the Joint Genome Institute, supported by HL066681 (LAP), Berkeley-PGA, under the Programs for Genomic Application, funded by National Heart, Lung, and Blood Institute, USA, was performed under Department of Energy Contract DE-AC02-05CH11231, University of California (LAP).

\section{References}

1 Torfs CP, Curry CJ, Bateson TF et al: A population-based study of congenital diaphragmatic hernia. Teratology 1992; 46: 555-565.

2 Cass DL: Fetal surgery for congenital diaphragmatic hernia: the North American experience. Semin Perinatol 2005; 29: 104-111.

3 Slavotinek AM: The genetics of congenital diaphragmatic hernia. Semin Perinatol 2005; 29: 77-85.

4 Pober BR, Lin A, Russell $\mathrm{M}$ et al: Infants with Bochdalek diaphragmatic hernia: sibling precurrence and monozygotic twin discordance in a hospital-based malformation surveillance program. Am J Med Genet A 2005; 138: 81-88.

5 Slavotinek A, Lee SS, Davis R et al: Fryns syndrome phenotype caused by chromosome microdeletions at $15 \mathrm{q} 26.2$ and $8 \mathrm{p} 23.1$. I Med Genet 2005; 42: 730-736.

6 Kantarci S, Casavant D, Prada C et al: Findings from aCGH in patients with congenital diaphragmatic hernia $(\mathrm{CDH})$ : a possible locus for Fryns syndrome. Am J Med Genet A 2006; 140: 17-23.

$7 \mathrm{Lu} \mathrm{JR}$, McKinsey TA, Xu H et al: FOG2, a heart- and brain-enriched cofactor for GATA transcription factors. Mol Cell Biol 1999; 19: $4495-4502$.

8 Tevosian SG, Deconinck AE, Cantor AB et al: FOG2: a novel GATA-family cofactor related to multitype zinc-finger proteins Friend of GATA-1 and U-shaped. Proc Natl Acad Sci USA 1999; 96: 950-955.

9 Tevosian SG, Deconinck AE, Tanaka M et al: FOG2, a cofactor for GATA transcription factors, is essential for heart morphogenesis and development of coronary vessels from epicardium. Cell 2000; 101: 729-739.

10 Crispino JD, Lodish MB, Thurberg BL et al: Proper coronary vascular development and heart morphogenesis depend on interaction of GATA-4 with FOG cofactors. Genes Dev 2001; 15: 839-844.

11 Ackerman KG, Herron BJ, Vargas SO et al: Fog2 is required for normal diaphragm and lung development in mice and humans. PLoS Genet 2005; 1: 58-65.

12 Soriano P: The PDGF alpha receptor is required for neural crest cell development and for normal patterning of the somites. Development 1997; 124: 2691-2700.
13 Tallquist MD, Soriano P: Cell autonomous requirement for PDGFRalpha in populations of cranial and cardiac neural crest cells. Development 2003; 130: 507-518.

14 Ding $\mathrm{H}, \mathrm{Wu} \mathrm{X}$, Bostrom $\mathrm{H}$ et al: A specific requirement for PDGF$\mathrm{C}$ in palate formation and PDGFR-alpha signaling. Nat Genet 2004; 36: 1111-1116.

$15 \mathrm{Xu} \mathrm{X}$, Bringas Jr P, Soriano P, Chai Y: PDGFR-alpha signaling is critical for tooth cusp and palate morphogenesis. Dev Dyn 2005; 232: $75-84$.

16 Sun T, Jayatilake D, Afink GB et al: A human YAC transgene rescues craniofacial and neural tube development in PDGFRalpha knockout mice and uncovers a role for PDGFRalpha in prenatal lung growth. Development 2000; 127: 4519-4529.

17 Slavotinek AM: Fryns syndrome: a review of the phenotype and diagnostic guidelines. Am J Med Genet A 2004; 124: 427-433.

18 Corless CL, Schroeder A, Griffith D et al: PDGFRÁ mutations in gastrointestinal stromal tumors: frequency, spectrum and in vitro sensitivity to imatinib. J Clin Oncol 2005; 23: 5357-5364.

19 Hirota S, Ohashi A, Nishida T et al: Gain-of-function mutations of platelet-derived growth factor receptor alpha gene in gastrointestinal stromal tumors. Gastroenterology 2003; 125: 660-667.

20 Clarke ID, Dirks PB: A human brain tumor-derived PDGFR-alpha deletion mutant is transforming. Oncogene 2003; 22: 722-733.

21 Rand V, Huang J, Stockwell T et al: Sequence survey of receptor tyrosine kinases reveals mutations in glioblastomas. Proc Natl Acad Sci USA 2005; 102: 14344-14349.

22 Cools J, Stover EH, Boulton CL et al: PKC412 overcomes resistance to imatinib in a murine model of FIP1L1-PDGFR $\alpha$ lpha-induced myeloproliferative disease. Cancer Cell 2003; 3: 459-469.

23 Joosten PH, Toepoel M, van Oosterhout D, Afink GB, van Zoelen EJ: A regulating element essential for PDGFRA transcription is recognized by neural tube defect-associated PRX homeobox transcription factors. Biochim Biophys Acta 2004; 1588: 254-260.

24 Zhu H, Wicker NJ, Volcik K et al: Promoter haplotype combinations for the human PDGFRA gene are associated with risk of neural tube defects. Mol Genet Metab 2004; 81: 127-132.

$25 \mathrm{Au}$ KS, Northrup H, Kirkpatrick TJ et al: Promotor genotype of the platelet-derived growth factor receptor-alpha gene shows population stratification but not association with spina bifida meningomyelocele. Am I Med Genet A 2005; 139: 194-198.

26 Slavotinek AM, Moshrefi A, Davis R et al: Array comparative genomic hybridization in patients with congenital diaphragmatic hernia: mapping of four $\mathrm{CDH}$-critical regions and sequencing of candidate genes at 15q26.1-15q26.2. Eur J Hum Genet 2006; 14: 999-1008.

27 Bladt F, Riethmacher D, Isenmann S, Aguzzi A, Birchmeier C: Essential role for the c-met receptor in the migration of myogenic precursor cells into the limb bud. Nature 2005; 376: 768-771

28 Svensson EC, Tufts RL, Polk CE, Leiden JM: Molecular cloning of FOG2: a modulator of transcription factor GATA-4 in cardiomyocytes. Proc Natl Acad Sci USA 1999; 96: 956-961.

29 Jay PY, Bielinska M, Erlich JM et al: Impaired mesenchymal cell function in Gata4 mutant mice leads to diaphragmatic hernias and primary lung defects. Dev Biol 2006; 301: 602-614.

30 Huggins GS, Bacani CJ, Boltax J, Aikawa R, Leiden JM: Friend of GATA 2 physically interacts with chicken ovalbumin upstream promoter-TF2 (COUP-TF2) and COUP-TF3 and represses COUPTF2-dependent activation of the atrial natriuretic factor promoter. J Biol Chem 2001; 276: 28029-28036.

31 Klaassens $M$, van Dooren $M$, Eussen $H J$ et al: Congenital diaphragmatic hernia and chromosome 15q26: determination of a candidate region by use of fluorescent in situ hybridization and array-based comparative genomic hybridization. Am J Hum Genet 2005; 76: 877-882.

32 Prontera P, Buldrini B, Aiello V et al: Trisomy 15 mosaicism owing to familial reciprocal translocation $t(1 ; 15)$ : implication for prenatal diagnosis. Prenat Diagn 2006; 26: 571-576. 